\title{
Parents toxiques, addictions et psychothérapie institutionnelle ${ }^{\text {斻 }}$
}

\author{
Toxic parents, addictions, and institutional psychotherapy \\ Nicolas Evzonas (Docteur qualifié aux fonctions de maître de conférences \\ en lettres grecques, en cours de formation en psychologie clinique, \\ psychopathologie et psychanalyse) $)^{\mathrm{a}, \mathrm{b}, *}$ \\ ${ }^{a}$ Université Paris-Sorbonne-Paris IV, I, rue Victor-Cousin, 75005 Paris, France \\ ${ }^{\text {b } U F R ~ d ' e ́ t u d e s ~ p s y c h a n a l y t i q u e s, ~ u n i v e r s i t e ́ ~ P a r i s ~ D i d e r o t-P a r i s ~ V I I, ~ 5, ~ r u e ~ T h o m a s-M a n n, ~} 75013$ Paris, France
}

Reçu le 6 novembre 2015

\begin{abstract}
Résumé
Objectifs. - À travers la restitution d'un récit clinique, nous tentons de saisir comment la prise compulsive de toxiques s'articule avec la défaillance de l'environnement familial. Nous essayons par ailleurs de démontrer la pertinence de la prise en charge des addictions chez les sujets psychotiques dans l'espace multiréférentiel de la psychothérapie institutionnelle.

Méthode. - L'analyse du cas de Léonard s'appuie sur les théories métapsychologiques et les principes pluridisciplinaires de la psychothérapie institutionnelle. Sont privilégiées les notions du Collectif et du transfert dissocié chez le sujet psychotique conceptualisées par J. Oury ; les concepts winnicottiens du miroir et de l'objet transitionnel ; les procédés autocalmants théorisés par l'École psychosomatique de Paris ; divers postulats plaidant contre la pureté des structures psychopathologiques (W.R. Bion, C. Chaperot, M. Czermak, R. Chemama, J.-D. Nasio).

Résultats. -L'observation de Léonard, qui évolue dans l'espace transversal et multitransférentiel de la psychothérapie institutionnelle depuis dix mois, tout en possédant à son actif de nombreuses années de psychothérapie dans un cadre duel, permet de déceler une immense carence affective et une animosité visà-vis de ses parents, toutes deux dissimulées derrière un récit policé idéalisant l'amour parental. S'il se montre toujours peu apte à élaborer ses sentiments envers ses géniteurs, le patient, sujet à des alcoolisations
\end{abstract}

\footnotetext{
ț Toute référence à cet article doit porter mention : Evzonas N. Parents toxiques, addictions et psychothérapie institutionnelle. Evol Psychiatr 2016;81(4): pages (pour la version papier) ou URL [date de consultation] (pour la version électronique).

* Auteur correspondant. 36-38, rue Saint-Paul, 75004 Paris, France.

Adresse e-mail : nicolas.evzonas@gmail.com
} 
massives et à tous types d'auto-intoxications, commence, de par son intégration dans le Collectif, à afficher une meilleure maîtrise de ses comportements compulsifs.

Discussion. - L'observation clinique à l'appui de l'anamnèse conduit à l'hypothèse d'un désinvestissement précoce du patient par sa mère, aggravé par la présence matérielle d'un père psychiquement absent. La prise de toxiques se dessine comme une automédication colmatant les failles narcissiques consécutives à cette double carence et comme un effort de faire sans l'autre. Par ailleurs, la coexistence de traits névrotiques et psychotiques chez Léonard nous incite à questionner le purisme de la psychopathologie structurale commune. Conclusions. - L'hypothèse diagnostique qui ressort de nos analyses est celle d'une psychose partielle dysthymique. Nous défendons la thèse selon laquelle cette structuration psychique de Léonard plonge ses racines dans le pacte inconscient de ses parents pour lui interdire de naître et d'exister psychiquement. Par ailleurs, les investissements transférentiels du sujet psychotique, capables de se diffracter et de se diriger indistinctement vers toute personne qui gravite autour de lui, ainsi que vers une foultitude d'objets, nous permettent de concevoir l'espace pluriel de la psychothérapie institutionnelle comme un lieu d'objets vivants susceptibles de prendre la place des objets toxiques non humains.

(c) 2016 Elsevier Masson SAS. Tous droits réservés.

Mots clés : Addiction ; Psychothérapie institutionnelle ; Psychose ; Transfert dissocié ; Mélancolie ; Miroir ; Mère morte ; Procédés autocalmants ; Objet transitionnel ; Structure psychique

\section{Abstract}

Aims. - The aim of this article is to present a clinical case in order to demonstrate how the compulsive use of toxic substances articulates with the shortcomings of the family environment. It also aims to show the pertinence of the treatment of addictions in psychotic patients within the multireferential setting of institutional psychotherapy.

Method. - The analysis of the case of Léonard is based on metapsychological theories and the pluridisciplinary principles of institutional psychotherapy. More specifically, the article centers on the following: the Collective, and dissociated transfer, conceptualized by J. Oury; the Freudian theory of identification, melancholy and mania; Winnicott's concepts of the mirror and the transitional objet; the Complex of the Dead Mother by A. Green; the self-calming techniques theorized by the Psychosomatic School of Paris; the function of toxic substances as being pharmakon, namely poison and remedy, propounded by S. Le Poulichet; and theories disputing the purity of psychopathological structures (W.R. Bion, C. Chaperot, M. Czermak, R. Chemama, J.-D. Nasio).

Results. - The institution where I met Leonard encourages the free circulation of patients, their active participation in their cure, and cross-disciplinary treatments with special emphasis on sociotherapy, and constant work on the atmosphere, analysis and exploitation of multifocal transfers specific to psychotic patients. This approach appears to be liable to reduce both psychological alienation and social estrangement. The observation of Leonard, who has been testing this setting for 10 months after having experienced numerous years of individual psychotherapy, evidences tremendous emotional deprivation and animosity towards his parents, hidden behind the polished narrative of an idealized parental love. The patient, who is prone to massive absorption of alcohol and to several other types of self-intoxication, although he still does not seem capable of elaborating his emotions towards his progenitors, is beginning to gain some control over his compulsive behaviours as a result of his integration into the Collective setting.

Discussion. - Clinical observation, backing up the anamnestic approach, leads to the hypothesis of an early withdrawal of the emotional involvement of Leonard's mother, who suffered from depression at the time of her son's birth, following the loss of her own father. This primitive disaffection most probably had disastrous consequences on her son's psyche, since a depressed mother is likely to be a psychologically "dead mother" (A. Green), a distorting reflection of her baby's internal image and a terrifying mirror of his destiny (W. Winnicott). The patient explains the calm that drugs provide him with, but refuses to admit that he derives any satisfaction from them, which suggests that the use of toxic substances is a self-calming strategy resembling the compulsive and repetitive movements or tics deployed by an infant whose mother is physically 
or psychologically unavailable and/or unwilling to satisfy his needs. The emotional abandonment of Leonard was probably compounded by a substantially absent father, preoccupied with success, money and his spouse's unfaithfulness. Suicide attempts with his father's painkillers or vehicle, along with the explicit perception of his father as toxic, could provide some understanding of Leonard's compulsive behaviour as being a means of expending his anger towards his father. Overall, the act of taking toxic substances appears as a form of selfmedication liable to fill the narcissistic gaps caused by this dual deprivation in his early family environment, an effort to "do without" the other. Moreover, the coexistence of neurotic and psychotic mechanisms in Leonard's mental function encourages debate on the existence of pure, unadulterated psychopathological structures.

Conclusion. - This suggests a diagnostic hypothesis of partial dysthymic psychosis, that is to say a mainly psychotic structure with elements of mania and melancholy, dominated by foreclosure and compounded by neurotic features caused by the mechanism of repression. I suggest that the structuring of the patient was heavily influenced by his parents' unconscious pact to forbid their son to be and to be born. Furthermore, the fragmented transference investments that psychotic patients tend to make without distinction towards any person who gravitates around them, as well as towards a wide range of objects, leads us to conceive the pluralistic setting of institutional psychotherapy as a setting of living objects able to pacify the internalized, noxious parents and replace the toxic, inhuman, fetishized objects.

(c) 2016 Elsevier Masson SAS. All rights reserved.

Keywords: Addiction; Institutional psychotherapy; Psychosis; Dissociated transference; Melancholy; Mirror; Dead mother; Self-calming strategies; Transitional object; Psychic structure

\section{Introduction}

La présente contribution est le fruit d'un stage que nous avons effectué dans le cadre de notre formation universitaire en psychologie clinique au sein d'une structure régie par les principes de la psychothérapie institutionnelle. Notre objectif est ici de réinterroger le lien, maintes fois débattu, entre comportements addictifs et environnement familial défaillant, à partir de notre expérience dans cette institution, qui possède pour particularité de mettre au service de la cure l'analyse du transfert multifocal, lequel correspond à la propension dissociative du sujet psychotique. Pour questionner ledit lien, on restituera de manière circonstanciée, mais anonymisée le récit clinique d'un jeune homme appelé Léonard ${ }^{1}$, victime de lourdes carences affectives depuis sa prime enfance et qui se présente aujourd'hui comme un toxicomane et un « risqueur ${ }^{2}$. En vérité, il s'agit de transmettre une histoire transférentielle vécue au fil de nos interactions avec le patient au sein de ce dispositif spécifique, dans le but d'appréhender la dimension inconsciente de ses symptômes et de démontrer la pertinence du traitement par la psychothérapie institutionnelle, une prise en charge collective, transversale et multiréférentielle.

Si nous admettons l'existence de fondements génétiques et/ou biologiques dans la genèse aussi bien des psychoses que des addictions, notre démonstration restera focalisée sur le rôle parental dans la « décompensation » de la structure et sera exclusivement psychanalytique. Dans

\footnotetext{
${ }^{1}$ Le choix de ce prénom nous a été dicté par certains éléments anamnestiques du patient renvoyant à l'ouvrage d'A. Green sur l'étude freudienne de Léonard de Vinci [1].

2 Néologisme avancé par É.-P. Toubiana [2] pour décrire la mise en œuvre d'un éprouvé lié au risque, qui cohabite avec la dépendance et la compulsion de répétition chez les sujets souffrant d'addiction.
} 
le cadre de cette lecture métapsychologique, nous reprendrons les thèses freudiennes classiques sur l'identification, la mélancolie et la manie pour aborder l'ambivalence de Léonard envers son père. Ensuite, pour interroger la relation du patient avec sa mère qui souffrait de dépression au moment de sa naissance, on s'appuiera sur la théorie de Winnicott sur le fonctionnement du visage maternel en tant que miroir symbolique, ainsi que sur le complexe de la mère - psychiquement - morte conceptualisé par A. Green. Les théories de l'École Psychosomatique de Paris sur les procédés autocalmants nous aideront par ailleurs à saisir la compulsion de répétition qui régit le comportement de notre patient, en la mettant en lien avec l'objet primaire carentiel et défaillant. Une relecture de la notion de l'objet transitionnel de Winnicott en relation avec la toxicomanie s'avérera également fructueuse. Enfin, nous nous pencherons sur la structure psychique de Léonard dont le caractère ambigu remet en question la psychopathologie structurale courante. En conclusion, nous reviendrons sur les particularités du dispositif qui nous a accueilli et nous a permis de constater qu'une problématique aussi polyfactorielle que l'addiction ne peut être abordée efficacement que dans un cadre plurifocal comme celui proposé par la psychothérapie institutionnelle.

Nonobstant, compte tenu de la courte durée de notre stage - dix mois - et de notre familiarisation récente avec la dimension orchestrale de cette psychiatrie dynamique, ouverte et tolérante, il convient de reconnaître les limites de nos postulats. Si notre expérience dans cet espace collectif nous a permis de constater que le transfert ne constitue pas l'apanage exclusif du setting analytique, tel qu'on le conçoit classiquement, elle nous incite par ailleurs à considérer avec circonspection et réserve les changements remarqués dans l'attitude psychique du patient, éventuellement imputables à un étayage plutôt qu'à des remaniements profonds issus de mouvements inconscients. Notre tentative de théorisation de la prise de toxiques comme plongeant ses racines dans les défaillances parentales gagnerait certainement en profondeur si un suivi prolongé du patient était envisageable et si la supervision de notre stage au sein de l'institution pouvait s'ouvrir à des soignants extérieurs au suivi psychothérapeutique de Léonard. En sus, il ne peut être exclu que notre approche ait subi l'effet contaminant d'une certaine idéalisation de la psychothérapie institutionnelle de la part de l'équipe soignante, défense « maniaque » contre le vécu mélancolique que le travail avec les patients psychotiques est susceptible de provoquer [3].

\section{Le Collectif ou la constellation d'une transversalité}

Il importe de commencer par une esquisse de l'institution qui nous a accueilli durant notre stage et une brève description des principes qui régissent son fonctionnement. Précisons de prime abord que le nouveau visiteur est immédiatement frappé par la démédicalisation du lieu et par l'effacement de la distinction soignants/soignés. La blouse blanche qui brille par son absence comme la fine connaissance des arcanes du centre, dont les patients qui s'empressent de servir de guide formateur au néophyte font preuve, soulignent bien le caractère singulier du dispositif. Celuici constitue l'un des bastions de la psychothérapie institutionnelle, au carrefour de la biologie, de la sociologie, de la politique, de l'anthropologie et de la psychanalyse. On y ressent rapidement l'optimisation du travail sur l'ambiance, à savoir la lutte contre les effets pathoplastiques ${ }^{3}$ du milieu institutionnel - entendu comme climat extérieur, tonalité interne et interactivité plurimodale d'une

\footnotetext{
3 J. Oury a repris le terme médical « pathoplastie » pour introduire une dimension pathogène directement en relation avec le milieu environnant. En fait, il s'agit pour lui de la composante réactionnelle au milieu. Sur cette notion, qu'il développe tout au long de son œuvre, voir plus particulièrement [3,4].
} 
foultitude d'informations dotées d'une micro-sémiotique inconsciente -, mais aussi la promotion de la «sous-jacence» atmosphérique comme outil thérapeutique ${ }^{4}$. La porosité identitaire des patients psychotiques ou borderline pris en charge au sein de cette structure semble potentialiser la réceptivité aux effets de ce traitement ambiantiel fait d'actes parlants et de jeux signifiants, véritable «psychodrame au quotidien ».

La salle d'accueil de l'institution, expression emblématique de l'ambiance et véritable lieu de passage, promeut l'échange entre les patients, les soignants et les stagiaires autour d'un quotidien partagé, ponctué de repas, de lectures, de musique et de fêtes, au pouvoir thérapeutique essentiel. Il s'agit pour chacun de conjuguer dans cet espace « l'être avec » le patient psychotique et de trouver une qualité de présence à l'autre en réduisant le bruit de fond et l'agitation. On soulignera le caractère informel de la salle d'accueil qui décentre les activités et les entretiens thérapeutiques, ainsi que l'hétérogénéité qui imprègne le lieu - plusieurs origines, langues et sexualités s'y confondent et s'y confrontent -, permettant à l'imprévu de surgir et constituant autant de points d'accroche où pourra s'arrimer le désir du sujet.

Étant donné que l' «aliénation transcendentale » due à la maladie définit pour le psychotique la difficulté à être sujet d'énonciation de son propre désir et que cette aliénation se double de l'aliénation sociale que chacun est à même de subir [7], le postulat qui préside à l'institution est que l'homme est un être désirant qu'il s'agit de réintroduire dans le langage et le symbolique par le biais d'activités qui font sens pour lui. Les nombreux ateliers d'expression et groupes thérapeutiques proposés au sein de la structure - gymnastique, jardinage, cuisine, lecture, écriture, théâtre, etc. - ont pour objectif d'aider les patients psychotiques à « recoller » l'image fracturée de leur corps, à rassembler leur éparpillement psychique, à mobiliser les interrelations entre eux et à les réintroduire dans le lien social.

Le club thérapeutique, composé de façon paritaire de soignants et de malades, possède également une fonction désaliénante et structurante. Fondé sur le principe du patient-acteur social et co-acteur du soin, ainsi que sur la valeur anthropologique accordée au travail, ce club implique l'endossement de responsabilités, la participation à des décisions collectives et à l'exécution de tâches variées, en fonction des compétences et des spécificités de chaque membre, afin de permettre à chacun de se réinscrire dans un champ symbolique où le sujet est convoqué au niveau de sa pathologie au sein d'un système d'échanges. Ce qui contribue à diminuer certains symptômes et à soigner l'ambiance de l'institution. C'est en ce sens que la sociothérapie travaille sur la psychopathologie $[7,8]$.

La multiplication des investissements et des médiations s'accorde avec la particularité, relevée par J. Oury, du transfert psychotique dissocié et plurifocal qui tolère mal la conflictualité découlant de la confrontation avec l'autre dans un cadre duel [7]. Cette «constellation transférentielle », toujours selon l'expression de J. Oury, suppose des réunions de constellations de tous ceux qui, gravitant autour d'un patient - et ce, indépendamment de leur statut -, peuvent être support d'un fragment transférentiel, bien souvent à leur insu ou à leur « corps défendant » [7]. Lors de ces réunions s'effectue une mise au point sur les identifications et contre-transferts de chacun face aux patients et un travail d'élaboration au niveau de l'équipe visant à un rassemblement psychique du sujet psychotique, à la précision ou à la nuance du regard ou du diagnostic porté sur lui. Ainsi sont amorcées une mise en acte d'une transversalité et une polyphonie de discours, de fonctions, d'espaces, d'investissements et de sous-jacences, qui forment cette « machine abstraite » traitant

\footnotetext{
${ }^{4}$ C. Chaperot ([5], p. 93-210), C. Chaperot et A. Altobelli ([6], p. 541-555) recourent au terme « sous-jacence » (employé par J. Oury au sens de la charge inconsciente du désir) pour souligner la dimension inconsciente de l'ambiance institutionnelle.
} 
l'aliénation chosifiante aussi bien psychotique et sociale, conceptualisée par J. Oury sous le vocable désormais incontournable de Collectif [9].

\section{Le cas Léonard ou l'histoire d'un rescapé miraculeux de la mort}

\subsection{Cadre institutionnel}

C'est dans cet espace vivant et grouillant de transferts entrecroisés que nous avons fait la connaissance de Léonard. Nous l'avons rencontré dans plusieurs groupes thérapeutiques que nous avons co-animés pendant notre stage. Nous avons eu également l'occasion d'échanger en duo avec lui dans l'effervescence de la salle d'accueil, dans la sérénité de la cour ou devant l'entrée du Centre, dans un restaurant pendant une fête organisée par le Collectif ou à l'orchestre d'un théâtre après sa prestation lors du spectacle exposant le travail mené dans les ateliers d'expression. En somme, nous avons pu interagir avec le patient aux interstices de l'institution et au creux des événements quotidiens et/ou exceptionnels qui constituent des opérateurs cruciaux de la psychothérapie institutionnelle. Nos interactions avec Léonard, qui s'inscrivent dans un « vivre ensemble » fondé à la fois sur un échange verbal et sur un partage d'objets (un repas, une cigarette, un livre, une photo, un film, etc.), si elles remettent en question la neutralité du thérapeute, laquelle peut d'ailleurs s'avérer très malveillante, voire mortifère dans la prise en charge des pathologies lourdes ${ }^{5}$, respectent bel et bien l'interdit fondamental de l'abstinence.

S'agissant de la justification de ce setting institutionnel privilégiant une autre forme de transfert que celle du travail analytique orthodoxe, nous faisons nôtres les propos exprimés par P. Faugeras : «La clinique des psychoses est d'une exigence telle qu'elle nécessite que les paradigmes fondamentaux qui déterminent notre être au monde, comme les relations à l'autre, à l'espace et au temps par exemple soient non seulement interrogés dans leur évidence mais encore que l'on accepte l'idée que cette clinique du sujet dissocié puisse avoir pour adresse multiplicité et dispersion, et, pour cadre une extraterritorialité qui semble s'étendre jusqu'aux limites du monde »[12].

\subsection{Anamnèse}

Quelques éléments de l'anamnèse du patient sont de mise à présent : Léonard est né au sein d'une famille fortunée, d'un père juriste et d'une mère évoluant dans le monde du spectacle. La mère, séductrice et alcoolique, avait commis quelques écarts extraconjugaux que son mari n'a jamais réussi à lui pardonner, ni même à évoquer avec son psychiatre avant le décès de son épouse. Elle-même avait perdu son propre père peu avant de mettre Léonard au monde. Elle est décédée au terme de plusieurs années de combat contre une grave maladie, son mari et Léonard à son chevet.

Léonard a ressenti très tôt sa différence et son étrangeté et a manifesté dès l'enfance des signes d'isolement et de repli. Pendant son adolescence, il s'est mis à boire, à fumer et à consommer du cannabis. Ce fut le début d'un long parcours de dépendance à des substances toxiques. À plusieurs reprises, il a également pris le volant en état d'ébriété et eu de graves accidents qui auraient pu se

\footnotetext{
5 On rappellera que S. Ferenczi avait déjà, en 1932, dénoncé l'attitude abstentionniste de l'analyste vis-à-vis des patients gravement traumatisés ([10], p. 126-128). P. Chemla a plus récemment exposé à quel point la neutralité du thérapeute peut être délétère dans la prise en charge des psychoses et des états-limite ([11], p. 43-59).
} 
révéler fatals. Ces conduites lui ont valu une série de condamnations pénales; nonobstant, grâce aux relations et à l'aisance financière de ses parents, il s'en est toujours tiré sans dommages.

Au cours de sa jeunesse pubertaire, Léonard a connu un épisode de bouffée délirante : il a imaginé que des étrangers avaient envahi un camping et enlevé tous les vacanciers tandis que lui avait failli être assassiné. Ce fut le point de départ d'un délire. À compter de ce moment, il a échafaudé un délire de persécution mettant en scène plusieurs ennemis : les extraterrestres, la mafia, les habitants de son village natal et ses parents.

Au cours des années suivantes, Léonard a été traité pour dépression dans le cabinet privé d'un psychanalyste. Dans le même temps, il a entamé plusieurs formations professionnelles sans jamais parvenir à les achever et exercé plusieurs métiers différents. Il a quitté le toit familial pour vivre seul et l'a réintégré quelques années plus tard pour des raisons financières, mais aussi pour accompagner sa mère pendant sa maladie. Incapable de supporter la cohabitation avec son père après la disparition de sa mère et n'ayant pas la possibilité d'assumer financièrement un logement personnel, il a demandé à être accueilli dans un foyer postcure où il réside actuellement. Or, lors d'un retour ponctuel dans la maison familiale, il s'est intoxiqué - tout comme il l'avait fait à la suite du décès de sa mère - en avalant massivement les antidouleurs de son père malade et il a dû être hospitalisé.

S'il bénéficie depuis plusieurs années d'un suivi psychiatrique et d'une psychothérapie au sein de l'institution, il n'accepte de participer aux ateliers thérapeutiques et de fréquenter le Collectif que depuis dix mois à peine. Sur le plan pharmacologique, il est traité avec un cocktail de neuroleptiques et de thymostabilisateurs. Enfin, à sa demande et dans le but de maîtriser ses excès éthyliques, son psychiatre lui a prescrit un médicament à effet antabuse.

\section{Le père toxique ou l'envers caché de l'idéal}

Dès la première séance de l'atelier thérapeutique dans lequel nous avons rencontré Léonard, son désir d'indépendance s'est dessiné comme un leitmotiv dans son discours. Ceci peut s'entendre naturellement comme le désir du sujet addicté de s'affranchir de son assujettissement au toxique, de cet esclavage aliénant suggéré par l'étymologie du terme « addiction » ${ }^{6}$. Les propos de Léonard ont, à maintes reprises, confirmé cette quête de liberté. Or, parallèlement à son envie de s'extraire de la spirale infernale de la toxicomanie, il a souligné plus d'une fois l'importance pour lui de trouver un logement personnel et de prendre son indépendance vis-à-vis de son père, sous le toit duquel il vivait après le décès de sa mère. Il est assez significatif que, se référant à cette cohabitation asphyxiante avec son géniteur, il ait formulé la phrase suivante : « on est devenu toxiques l'un pour l'autre ». Cette toxicité réciproque doit certainement être associée à la difficulté pour le père de supporter les addictions de son fils (comme Léonard lui-même l'a avoué), et à la difficulté pour le fils de supporter ce rejet. Outre ce sens métaphorique, elle doit suggérer par ailleurs l'interdépendance entre la relation père/fils et le choix du toxique comme objet fixe dans l'économie libidinale de Léonard. Est-il besoin de rappeler que le patient s'est intoxiqué à deux reprises en avalant en overdose les antalgiques de son père ? Ou que, sous l'emprise de l'alcool, il

\footnotetext{
${ }^{6}$ Quoique ce terme ait longtemps été considéré comme un anglicisme dérivé du verbe anglais addict, le mot «addiction» possède en vérité une racine latine. Ad-dictus comporte la double idée de contrainte de corps et de dette. Les esclaves romains étaient ainsi des « addictés »; privés de liberté et dénués de nom propre, ils étaient littéralement « dits par » leur maître, au sens strict d'aliénation. Dans le droit médiéval, l'addiction signifie qu'un débiteur est « contraint par corps », dès lors qu'il ne peut s'acquitter de sa dette. Le créancier avait ainsi le droit de saisir la personne du débiteur, il pouvait disposer du corps et de la personne $[13,14]$.
} 
s'est emparé du véhicule de ce dernier pour aller s'écraser contre un mur ? Ou encore que lorsque le foyer de postcure a accepté de l'héberger, ce qui impliquait une séparation au «scalpel » de son père, il s'est retrouvé à l'hôpital après une alcoolisation massive ?

Il est frappant de constater que Léonard n'est pas du tout conscient des sentiments négatifs qu'il nourrit à l'égard de son père, à en juger par les rationalisations constantes avec lesquels il le protège : s'ils se disputent, c'est parce que la cohabitation au quotidien génère inévitablement des frictions ; s'il a ingéré les comprimés de son père, c'est parce qu'il était malheureux à cause du décès de sa mère ou parce qu'il s'est éloigné du cocon apaisant du foyer et non parce qu'il s'est retrouvé seul avec son père dans la maison familiale. S'il est obsédé par son échec professionnel, ce n'est pas parce que son père lui a mis la pression concernant ses choix de métier, c'est parce que la société le malmène par l'exaltation de la réussite. En somme, chaque fois que nous introduisions discrètement une problématique susceptible de mettre en péril l'image d'un père irréprochable, Léonard réagissait par une anathématisation d'un facteur extérieur et un surenchérissement sur l'amour paternel.

On insistera sur le fait que le père de Léonard est esquissé dans le discours explicite de son fils non seulement comme un tendre tuteur mais aussi comme un idéal absolu : représentation de la brillance professionnelle, du rayonnement social et de l'érudition. Nous avons trouvé intrigant le fait que, lorsque nous avons un jour initié une discussion autour d'un mythe antique au sein d'un atelier, Léonard a fait un commentaire passionnant sur l'appropriation de cette légende par un artiste contemporain et lorsque nous lui avons fait remarquer combien sa contribution était intéressante, il a tenu à rappeler que ses connaissances étaient rudimentaires, à la différence de celles de son père, si cultivé. En d'autres occasions, il a exalté la générosité sans bornes de son géniteur et le fait que, grâce à lui, il n'avait jamais manqué de rien sur le plan financier, laissant simultanément transparaître en négatif d'autres types de carence.

D'une façon similaire, il exprimait ses égards envers son psychiatre, voire l'admiration éperdue qu'il lui vouait, pour le dénigrer subrepticement dans la foulée. L'idéalisation se dessinait petit à petit dans le discours de Léonard comme une défense contre l'agressivité envers la figure paternelle, comme un paravent faisant écran à l'image d'un parent psychiquement absent, accaparé par l'appât du profit, l'ambition sociale et d'autres soucis. Grâce aux récits croisés que nous avons pu écouter pendant les réunions de constellations auxquelles le psychiatre du père de Léonard participait, nous avons compris que ledit patriarche était rongé par l'infidélité de son épouse depuis avant la naissance de Léonard, ce qui peut expliquer - au moins en partie - le désinvestissement de son rôle parental.

Force est de constater que, en dépit de multiples années de psychothérapie, Léonard demeure incapable d'exprimer sa colère envers son père. Au lieu de traiter psychiquement le conflit qui l'oppose à lui, il maintient comme clivée l'image d'un géniteur magnifié et celle d'un tuteur exécrable, ce qui l'amène à liquider sa rage par des voies externes courtes, autrement dit par les comportements et l'agir : la prise de toxiques, dont la valeur symbolique de «stupéfaction » de la douleur psychique se concrétise par l'ingestion des antalgiques paternels, c'est-à-dire de stupéfiants censés agir sur la douleur physique. Dans une logique qui confond les registres du somatique et du psychique, ou peut-être encore, du littéral et du métaphorique, l'antidouleur n'est-il pas ce que l'on avale pour ne plus souffrir ?

\section{Le délire de petitesse ou le triomphe maniaque contre l'objet paternel mélancolisé}

Un motif solidaire du thème de l'idéalisation, qui a émergé lors de nos échanges avec Léonard, est sa grande mésestime de soi. Le jeune homme se décrivait comme un raté et comme un vaurien 
qui n'avait entrepris aucun cursus d'études supérieures, n'avait exercé nul métier prestigieux et ne possédait aucune culture, à l'inverse de son père ou de nous qui avions accumulé de nombreux diplômes. Dans ses propos, nous voyions se profiler en filigrane l'image d'un moi avili et méprisé au profit d'un autre idéalisé qu'il projetait sur son père, son psychiatre ou sur nous. Il savait justifier ses arguments en s'appuyant astucieusement sur certains relia, cependant, nous nous sommes vite aperçu que plusieurs de ses affirmations ne reposaient sur aucun véritable fondement. Souvent d'ailleurs, il nous surprenait par ses remarques pertinentes et ses références artistiques ou autres, alors même qu'il était persuadé de débiter des platitudes. Il n'arrêtait pas de se reprocher l'état de sa santé mentale, la honte qu'il représentait pour sa famille, la tentative de suicide de son père. Son discours prenait souvent une tonalité d'auto-tourment et d'une véritable rage envers soi.

$\mathrm{Au}$ vu de tout ce qui a été signalé plus haut par rapport au père de Léonard, on ne peut s'empêcher de penser à l'hypothèse freudienne de l'identification à l'autre dissimulée derrière les autoreproches. Dans cette optique, la dévaluation constante de soi et l'autoagressivité, voire l'autoagression concrétisée par les intoxications volontaires et les conduites automobiles suicidaires via des objets appartenant au père (antalgiques, voiture), pourraient être comprises comme une attaque forcenée envers une figure paternelle vexante et décevante. Si l'on prend en considération par ailleurs le désir de Léonard d'acquérir son indépendance vis-à-vis de son père (géographiquement, financièrement et, plus implicitement, psychiquement), l'on pourrait concevoir ses passages à l'acte comme un triomphe maniaque contre l'objet paternel mélancolisé. Rappelons que Freud a soutenu que la manie constitue une victoire vis-à-vis de l'objet perdu, qui phagocyte le mélancolique et abrase son Moi, et que le maniaque, qui ressemble à l'alcoolique, nous démontre, « en partant comme un affamé en quête de nouveaux investissements d'objet, qu'il est libéré de l'objet qui l'avait souffrir » [15]. Ce qui incite P.-L. Assoun à extrapoler que l'abonné aux toxiques, à l'instar du mélancolico-maniaque, « célèbre l'indépendance [...] et se sent "libre comme l'air" au fond de sa geôle » [14].

Dans une perspective différente, on pourra envisager l'autodépréciation de Léonard et son pendant de surestimation paternelle, comme un moyen de se rapprocher d'un idéal. S'il se sent « nul », ériger son père en modèle constitue une façon de restaurer son narcissisme blessé, à la manière d'un admirateur éperdu, qui, au travers du culte qu'il voue à une star rayonnante, cherche à capter une partie de sa lumière. La magnification de l'autre ne constitue en vérité qu'une ruse du narcissisme. Écoutons à cet égard G. Benedetti : «Dans la mélancolie, la technique utilisée par le Moi dévalorisé pour se revaloriser est l'identification avec le Surmoi. [...] Le Moi tente de se revaloriser en s'auto-accusant. [...] En s'accusant, il s'identifie avec le Surmoi et tente de se revaloriser, parce qu'en soulignant ses innombrables fautes, il se place en dehors et au-dessus de celles-ci » [16]. En somme, l'autodénigrement de Léonard, pendant de l'idéalisation du père, constituerait une opération de renarcissisation et d'auto-exaltation.

\section{Le miroir de la «mère morte » ou la compulsion autocalmante}

Si le personnage du père occupe une place dominante dans le discours de Léonard, celui de la mère, quoique plus discrètement présent, nous aidera à concevoir plus justement la réalité psychique du patient en question et à mieux éclairer les « épousailles » maniaques avec l'objet toxique qui constituent l'enveloppe formelle de son malaise.

Léonard a mentionné qu'il rêvait souvent de sa maman, ce qui peut aisément se comprendre, compte tenu de son décès récent. Nonobstant, un morceau de rêve qu'il nous a raconté mérite une attention particulière. Il se souvient : «Ma mère est en train de conduire une voiture et moi je passe à côté d'elle, mais elle ne me voit pas ; je suis comme invisible ». Entendant ce petit 
fragment, nous n'avons pu nous empêcher d'établir un lien avec la question surprenante qu'il nous avait posé un autre jour : "Est-ce qu'on voit la mère des héros dans les dessins animés de votre pays? Car dans ce que je regardais quand j'étais petit à la télé française, y avait pas de mère »! » Une autre confidence souligne a contrario son sentiment d'abandon : "Le cancer de ma mère a été une épreuve pour toute la famille, mais moi j'étais content de veiller à son chevet et de pouvoir enfin être auprès d'elle ». Une mère qui « conduit » sa vie sans regarder son enfant, qui est complètement absente lorsque son fils a le plus besoin d'elle et qui parvient à se présentifier uniquement par son corps alité et mourant : voilà la figure maternelle qui se dessine en pointillé dans les récits de ce cancre autodestructeur qui calque son attitude sur celle des cellules cancéreuses de sa génitrice retournées contre leur hôte.

Sur ce point, on fera appel à certains concepts propres à ouvrir des espaces d' " intelligence des phénomènes », comme l'écrit $\mathrm{C}$. Chaperot, qui conçoit les théories comme « des paysages qui s'ouvrent, dans lesquels il devient plus simple de cheminer avec les patients, sachant que l'essentiel reste de descendre dans leur paysage à eux » [3]. En premier lieu, on invoquera Winnicott, qui conçoit le visage maternel comme un miroir symbolique, précurseur de tout miroir à la surface plane et réfléchissante, capable de refléter les états internes du bébé et de lui donner ainsi le sentiment d'exister ou, à l'inverse, en cas de dépression de la mère, de créer l'absence et le vide, nés de la discordance entre ce qu'il éprouve et ce qu'il contemple sur le miroir [17]. Les ravages qui s'ensuivent dans l'identité subjective du nourrisson, lorsqu'il subit le versant déstructurant et désymboligène de l'expérience du miroir primaire, sont proches de ceux découlant de l'identification à la mère endeuillée et psychiquement absente, conceptualisée par A. Green sous l'appellation du « complexe de la mère morte ». Dépressions blanches, trous psychiques comblés par des réinvestissements empreints de destructivité, dégâts sur l'avenir libidinal objectal et narcissique : voilà les effets du miroir médusant de la mère morte [18].

Ces théories nous aident à saisir de manière globale la faille originaire qui a dû survenir dans la structuration de Léonard par un défaut de holding, à cause d'une génitrice absorbée par son deuil personnel et globalement désinvestie de son rôle de mère. Elles ne nous éclairent pas pour autant sur le lien entre cette défaillance maternelle primaire et le destin pulsionnel particulier de Léonard, à savoir sa transformation en " esclave de quantité », pour reprendre la formule de M. de M'Uzan, qui définit ainsi l'ensemble de comportements soumis au besoin irrépressible de la recherche répétitive de l'excitation [19].

C'est ici que les théories des psychosomaticiens sur les «procédés autocalmants » peuvent s'avérer très fructueuses pour la compréhension de notre cas. Rappelons tout de même d'abord quelques données cliniques qui nous ont incité à nous tourner vers ces théories. Léonard a mentionné que ce qui constitue pour lui une invitation irrésistible à se droguer, c'est l' « ennui », que l'on peut entendre non seulement au sens de désœuvrement mais également au sens étymologique de torture, puisqu'il a avoué en d'autres occasions qu'il se sent « tourmenté par une cause inconnue et non identifiée », par un « vide sidéral » qu'il cherche à combler, par une « excitation qui vient d'ailleurs ». Il a confessé en outre que sa main, comme dissociée du reste de son corps, se tend vers les toxiques, lorsqu'il reste sans personne auprès de lui et qu'il se sent envahi par une « solitude incommensurable». Quand nous lui avons demandé si la consommation de stupéfiants lui procurait une euphorie ou une jouissance, il nous a répondu : « Non, ça me calme tout simplement !».

Cette dernière déclaration nous ramène vers l'opposition établie par M. Fain entre le satisfaisant, qui assouvit la revendication pulsionnelle, et le calmant, qui apaise l'excitation mais ne procure aucun plaisir [20]. Les procédés autocalmants suggèrent déjà par leur nom que l'objet est disqualifié au profit de l'autostimulation. G. Szwec introduit à cet égard la notion d' « anobjectalisation », qui définit un défaut de liaison avec le masochisme primaire et implique 
l'autonomisation vis-à-vis d'un objet primaire carentiel et défaillant, incitant à l'autobercement et à l'autogratification [21]. La compulsion de répétition qui s'y rattache (coups rythmiques, balancements, tics, etc.) constitue un effort de maîtrise du traumatique débordement excitationnel consécutif à la perte de l'objet. En conséquence, faute d'un étayage précoce du nourrisson par la mère (ou son tenant lieu), se mettent en place les techniques autocalmantes qui contre-investissent les représentations liées à la pulsion, surinvestissent le corps et les traces motrices, court-circuitent le processus de symbolisation par le recours aux voies courtes externes et promeuvent une activité masturbatoire mécanique, privée de support fantasmatique. En termes winnicottiens, on parlerait d'une faillite de la transitionnalité et d'une fétichisation/concrétisation de l'objet transitionnel [22,23]. Dans cette optique, l'addiction à l'objet toxique serait le fruit d'une fixation sur les aspects non vivants de l'aire transitionnelle et un leurre calmant renvoyant à un problème de séparation d'avec la mère non surmontée psychiquement en raison d'une défaillance du cadre familial. Selon une autre théorie, l'addiction peut être comparée à l'attachement à des objets non humains, souvent rencontré chez des sujets psychotiques ou limites, " qui tient à leur caractère constant, face à l'imprévisibilité et à l'effet traumatique des éléments humains de l'environnement » [23].

À la lumière de ces théories, on pourrait envisager que la vie agitée de la mère de Léonard, ses priorités artistiques, ses préoccupations extraconjugales, sa mésentente avec son époux, ses dérives éthyliques et le deuil de son père concomitant à la naissance de son fils ne lui aient pas permis d'assurer une présence câline, vivante et vivifiante auprès de son fils, de devenir son 《 jumeau imaginaire $»^{7}$ ou « double gémellaire ${ }^{8}$ structurant. Léonard a sans doute organisé un pare-excitations autonome de qualité douteuse et cultivé des perceptions fétichisées afin de colmater le vécu traumatique de la «mère morte », en difficulté de représentation. Ces perceptions ont dû constituer le noyau de son futur comportement répétitif autocalmant. La chronicisation de la situation de « vide sidéral », d' « ennui », de « solitude », de « dépression » et d'addictions calmantes suggère l'impact du « traumatisme cumulatif » [26] et l'absence d'influences compensatrices. Le rôle du père, décisif dans ce type de rattrapage psychique ${ }^{9}$, a également failli, comme nous l'avons vu plus haut. En définitive, le cas Léonard plaide ardemment en faveur de l'hypothèse freudienne ${ }^{10}$ selon laquelle « l'intoxication est un effort de faire sans l'autre » [28], opération qui peut être assimilée à la « machine à câlin » inventé par Temple Grandin pour mettre les personnes autistes en situation de contrôle et leur permettre de se prémunir des effets traumatiques des éléments humains de l'environnement.

\section{7. «Le roi des gardes-fous » ou une forclusion partielle du Nom-du-père}

Puisque les géniteurs de Léonard, accaparés par leurs histoires de couple et leurs autres soucis personnels, ont désinvesti leur rôle parental en se contentant d'assurer une présence matérielle auprès de leur fils, l'on comprend mieux pourquoi ils se sont « agrégés » dans le délire persécutif de

\footnotetext{
${ }^{7}$ Expression utilisée par D. Anzieu pour A. Green qui lui rend hommage en s'appropriant celle-ci dans un texte commentant le «testament spirituel » d'Anzieu : Le Penser, Du Moi-peau au Moi pensant [24].

${ }^{8}$ Cette expression, qui appartient à R. Roussillon, est utilisée par O. Lesourne ([25], p. 184) dans le contexte de la structuration psychique du futur toxicomane.

${ }^{9}$ C'est le mérite de certaines approches contemporaines [23,25] que d'avoir souligné la possibilité d'amortissement des traumatismes précoces liés à la faillite de la mère, via la présence réconfortante du père et/ou celle d'autres personnes importantes du milieu familial.

10 Dans sa lettre à Fliess du 22 décembre 1897, Freud a écrit que le besoin d'alcool, de morphine ou de tabac n'est que le substitut du « besoin primitif » de masturbation ([27], p. 211-212).
} 
Léonard, aux étrangers (Gitans, mafia) et autres créatures extraterrestres qui peuplaient son délire adolescent, échafaudé à partir de son unique hallucination. Leur participation au complot en vue de l'enlèvement et du meurtre de Léonard, à l'époque de sa dépression post-pubertaire, suggère leur fonction aliénante et extranéisante, ainsi que la déréliction du fils, le naufrage de toute confiance en eux et l'agressivité, voire la haine meurtrière à leur égard - mécanisme de projection à l'appui. Leur absence, leur silence et leur déni de sa souffrance, leurs interventions visant à gommer les conséquences légales de ses dérapages sans jamais poser de limites à son comportement, leur complicité envers ses vices addictifs (ils lui procuraient du cannabis) et l'autorisation implicite de « jouir » sans entrave aucune, ont créé un terrain propice à l'éclosion d'une pathologie qui interroge puissamment la question de l'existence ou de l'absence du Surmoi.

Signalons tout d'abord combien Léonard est attaché aux activités du Collectif et au cadre dans lequel celles-ci se déroulent. Il nous a expliqué sans ambages que la régularité et les horaires fixes des ateliers hebdomadaires, auxquels il participe assidument, constituent une invitation à la discipline et un garant de créativité pour lui, car l'existence de ces règles l'empêche de dévier vers ses addictions. «Le problème avec moi, c'est que je m'autorise trop de choses », nous a-t-il un jour avoué, exprimant sa difficulté à se poser des limites. C'est d'ailleurs la raison pour laquelle il n'a pas suivi d'études supérieures car « la fac donne trop de liberté ». Il nous a enuméré par la suite une série de précautions qu'il avait prises pour ne pas s'égarer dans une permissivité susceptible de le détruire, comme par exemple son initiative de confier son budget à un curateur pour se priver de la possibilité d'acheter des stupéfiants. Et, achevant son discours, il a prononcé cette phrase hautement significative : «Je suis le roi des garde-fous ». Léonard exprime on ne peut plus clairement son besoin vital d'être protégé par des gardiens externes afin de se préserver de la folie, c'est-à-dire afin de se prémunir contre cette jouissance sans bornes du Ça, qui n'est régulée par aucune barrière interne. Errant dans un No-Limits Land, il ne cesse d'ailleurs de répéter son «besoin de repères », que l'on ne peut s'empêcher d'entendre comme le besoin d'un père détenteur des règles qu'il n'a pu «psychiser ».

Quant à sa perception comme « roi » (des garde-fous), on devinera son désir d'être le roi de quelque chose, lui qui se compare avec ses parents de rang (social et professionnel) « royal » et qui se trouve «nul ». Être le roi, renvoie en outre à une toute-puissance mégalomaniaque, séquelle d'un narcissisme primaire, qui coexiste fréquemment avec le délire de petitesse propre au complexe mélancolique. Il s'agirait d'une oscillation entre le Moi merveilleux et le Moidéchet, sous-tendu par la logique du tout ou rien et expression de la «maladie d'idéalité » dont parle J. Chasseguet-Smirgel [29]. Léonard manifeste les symptômes de cette maladie lorsqu'il affirme être seul responsable de la tentative de suicide de son père (que l'on peut traduire par : je suis capable de conduire mon père à la mort parce que je représente son sens de vie $v s$ je suis un «parricide » méprisant) ou lorsqu'il se vante, l'air exalté, des innombrables formations professionnelles qu'il a suivies, pour confesser sur un ton morose qu'il n'a jamais pu en mener une seule à bien. En une autre occasion, il a mentionné que lorsqu'il joue de son instrument de musique, il se sent comme le Dieu incarné, alors que, quand nous l'avons croisé après sa prestation dans le cadre d'un spectacle organisé par le Collectif, il était dévasté à cause de sa performance qu'il jugeait «nullissime ». Si l'on intègre cette donnée dans le contexte de ses passages à l'acte dipsomaniaques, l'on peut se demander si les «noces » maniaques de Léonard avec l'objet toxique ne constituent pas une euphorisation instantanée de son Moi-déchet et un mariage furtif avec son Moi idéal ${ }^{11}$.

\footnotetext{
11 On renverra à cet égard à l'étude de P.-L. Assoun qui développe le lien entre l'alcoolisation et l'idéal ([14], p. 62).
} 
Il importe à présent de mentionner un « garde-fou » particulier : Léonard a exigé que son psychiatre lui prescrive un médicament à effet antabuse afin de maîtriser sa dipsomanie. La molécule qu'il a demandée provoque des nausées, des vomissements, des vertiges et un malaise, effets recherchés pour associer une sensation de dégoût à l'absorption de l'alcool. Au plan symbolique, cette drogue revêt la signification d'un gendarme rappelant à l'ordre le sujet défaillant. En fait, il s'agit d'un traitement « comportementaliste » qui scotomise toute dimension inconsciente du symptôme. Ce qui nous intéresse cependant ici, c'est de souligner que notre patient cherche désespérément à l'extérieur, ce qui lui fait défaut à l'intérieur, en l'occurrence une instance non seulement régulatrice, que le psychiatre ou l'analyste pourraient incarner, mais aussi sadique et punitive, sans doute en miroir de ses pulsions (loi du Talion).

Tout mène à penser que Léonard a échoué à constituer une barrière contre la jouissance illimitée du Ça et à former un Surmoi pacifiant, issu des identifications parentales œdipiennes bisexuées. Le procès constant qu'il se fait à lui-même, son autodénigrement, ses comportements masochistes et ses conduites suicidaires témoignent d'une malformation du Surmoi ou plutôt de l'existence d'un Surmoi archaïque, cruel, non humanisé, tel celui décrit par M. Klein [30] ${ }^{12}$. Le Surmoi de Léonard semble représenter une «pure culture de la pulsion de mort », pour emprunter une formule de Freud [32], ou le porte-parole d'un « narcissisme de mort », pour suivre le lexique d'A. Green [33]. En termes lacaniens, on parlerait de la manifestation d'un Moi idéal de toute-puissance maniaque échouant à évoluer vers un Idéal du Moi qui impliquerait la dette, le défaut, soit la castration symbolique et la tiercéisation, autrement dit l'introjection du Nom-du-Père $[34,35]^{13}$.

Nos dernières réflexions incitent à nous interroger sur le diagnostic de la pathologie de Léonard. S'agissant de patients souffrant d'addictions sévères, la doxa psychanalytique parle un peu trop rapidement de structuration (ou plutôt d'a-structuration) limite, comme si un symptôme, quelle que soit son ampleur, suffisait pour définir une structure par définition composée d'un ensemble d'éléments. On s'abstiendra ici d'entrer dans le grand débat autour de l'existence de la catégorie borderline considérée par de nombreux analystes comme une entité fictive, née d'une méconnaissance du transfert [36]. Rappelons plutôt ce que P.-L. Assoun écrit au sujet du lien entre l'addiction et la nosographie analytique :

« Il n'y a pas lieu d'attribuer à la toxicomanie la consistance d'une structure inconsciente sui generis. L'opération toxicomaniaque est en revanche à situer comme une réponse à une conjoncture traumatique et conflictuelle qui en retour interroge le rapport à la névrose, à la psychose et, sans doute électivement, à la perversion » [14].

Il poursuit en expliquant que l'addiction pourrait revêtir, selon les cas, la vocation d'un décuplement de la jouissance perverse, d'une « aération » du refoulement névrotique, d'une induction physique du montage délirant ou, au contraire, de l'écrasement du délire et des voix envahissantes dans la psychose [14]. Dans cette optique, le vide et l'étrangeté de sensations teintée de persécution, qui assaillent Léonard, pourraient être conçus comme des angoisses psychotiques

\footnotetext{
${ }^{12}$ Comme le résume bien J.-L. Donnet [31], « la description métapsychologique détaillée de la genèse et de l'évolution du Surmoi impose la conception d'un processus de surmoïsation très précocement à l'œuvre et impliquant des remaniements identificatoires permanents, modifiant le statut même des identifications, pour les rendre moins narcissiques et plus symboliques. C'est ainsi qu'à un registre surmö̈que précoce (Klein) reposant sur des mécanismes archaïques (identification à l'agresseur, talion), s'oppose un registre franchement postœedipien, où le Surmoi bisexué (constitué de deux identifications - paternelle et maternelle - est marquée par des élaborations psychiques subtiles [...]. » (souligné par nous).

13 C. Chaperot ([5], p. 80-86) montre très précisément, par des exemples cliniques, la fonction et la relation entre le Moi idéal et l'Idéal du Moi, conformément à leur conception par Lacan.
} 
sur lesquelles les toxiques agiraient comme un véritable pharmakon, selon la théorie de S. Le Poulichet, c'est-à-dire comme un remède qui constitue en même temps un poison [37].

La réflexion d'Assoun s'approfondit à notre sens, si l'on se retourne vers le fonctionnement global du patient pour essayer d'y repérer, indépendamment de sa symptomatologie, certains critères différentiels de sa structure inconsciente. Puisque la névrose implique avant tout l'existence d'un inconscient issu du processus de refoulement, il convient de s'interroger : Léonard a-t-il manifesté le sentiment subjectif d'une Autre scène ? Si l'on prend en considération le fait qu'il attribue systématiquement l'origine de son malaise à une cause extérieure - comme, par exemple, les altérations de son cerveau par la chimie des drogues -, sans être en mesure de conflictualiser sa relation avec ses parents ni de questionner sa division en Moi conscient et Moi inconscient, on sera enclin à exclure la pathologie névrotique. Quant à l'absence de différenciation entre le Moi idéal et l'Idéal du Moi, que nous avons relevée plus haut, et à la faillite de l'intégration du « Non-du-Père », qui ordonne et qui interdit - autre façon de définir la forclusion du Nom-du-Père [38] -, elles conduisent inéluctablement vers un diagnostic de psychose ${ }^{14}$.

Par ailleurs, si le discours de Léonard semble lisse, cohérent et bien mené, l'on notera son inaptitude spontanée à l'interprétation métaphorique de ses propos et à la recherche du signifiant éludé appartenant au registre inconscient. Les exemples du «père toxique », du « garde-fou » et des « antidouleurs », signalés auparavant, indiquent vraisemblablement une absence d'effet de signification, c'est-à-dire une absence de double sens repéré par Léonard au sein de son propre discours, ce qui pourrait faire penser à la signification psychotique qui renvoie à elle-même.

Que peut-on dire à présent des rêves de Léonard, de ses lapsus, de ses actes manqués, de ses fous rires, de ses mots d'esprit, tous ces signes d'un inconscient vivant faisant partie de $\operatorname{soi}^{15}$ ? C. Chaperot, qui admet que toutes ces manifestations « névrotiformes » de l'Autre scène sont légion chez les patients psychotiques, s'efforce de démontrer leur caractère factice, toute en plaidant pour l'existence d'une « orientation principale de la structure » [3] ${ }^{16}$, qui fait écho à la thèse bionienne de la partie psychotique et de la partie non psychotique de la personnalité [39]. M. Czermak, pour sa part, évoquant le registre du « tout ou rien » dans lequel se joue le mécanisme psychotique de la forclusion, émet simultanément des doutes quant à sa portée généraliste lorsque celle-là est en jeu [40]. Inspiré par Czermak, R. Chemama, quant à lui, introduit l'hypothèse de la «forclusion partielle » qui sous-tendrait le fonctionnement d'un sujet psychotique capable de recourir aussi à des mécanismes névrotiques [41]. J.-D. Nasio va encore plus loin : faisant de la forclusion l'affaire possible de tous, il élabore la notion de «forclusion locale », qui implique, selon lui, la scotomisation d'un traumatisme « n'affectant qu'un feuillet parmi les différents feuillets qui composent le Moi » et ayant pour résultat une folie « sociocompatible » [42].

Ces auteurs ont le mérite d'avoir relevé dans leur clinique certaines réalités psychiques mixtes à contre-courant de la psychopathologie structurale commune et d'avoir ouvert un débat fructueux sur la plasticité du psychisme. Dans la même veine, on sera enclin à soutenir l'existence chez Léonard d'une structure à dominance psychotique et d'une forclusion partielle du Nom-du-Père. Compte tenu des éléments mélancoliques et maniaques que nous avons pu discerner dans son

\footnotetext{
${ }^{14}$ Selon C. Chaperot ([5], p. 81), qui s'appuie sur sa riche expérience clinique, ainsi que sur les travaux de plusieurs spécialistes de la schizophrénie, « Moi idéal et Idéal du Moi constituent des instances typiquement névrotiques dans leur distinction, ces instances étant confondues dans la psychose, ou bien absentes ».

15 À distinguer de l'inconscient persécuteur du sujet psychotique, qui n'est pas subjectivement intégré par lui et qui s'autonomise de sa réalité interne.

${ }^{16}$ C. Chaperot ([5], p. 101) adhère également à l'idée d'une « partielle forclusion du complexe de castration », défendue par Lacan dans son Séminaire VI, le Désir et son interprétation.
} 
fonctionnement, l'on pourrait compléter cette hypothèse diagnostique et s'exprimer en termes de psychose partielle dysthymique.

\section{Conclusion}

Il nous paraît important d'aborder ici pour conclure les effets de la psychothérapie institutionnelle sur Léonard. Léonard a accompli un considérable pas en avant lorsqu'il a décidé d'adhérer à l'espace du Collectif. En effet, même s'il est suivi par un psychiatre et une psychologue depuis de nombreuses années, cela fait seulement dix mois qu'il participe aux activités collectives et qu'il bénéficie des effets symbolisants et socialisants du traitement ambiantiel de l'institution. On ne saurait mettre en doute l'évolution de Léonard consécutive à la prise en charge en « relation duelle » : c'est grâce à ce travail préalable de longue haleine qu'il a pu réussir à sortir du déni complet de sa maladie mentale - lequel ne faisait en vérité que reproduire l'attitude de ses parents - et assumer l'idée d'un traitement psychothérapique dans un espace peuplé d'autres patients gravement atteints, miroir implacable de sa propre souffrance.

Néanmoins, c'est à dater de son intégration dans le Collectif qu'il a commencé à expérimenter les bienfaits d'une thérapie globale et holistique qui lui a injecté un nouveau sens de vie. C'est grâce au support de la fiction au sein de l'atelier de poésie qu'il a pu élaborer discrètement et par bribes son ancien délire; c'est par la médiation de l'écriture dans un autre groupe qu'il a pu mettre en mots des choses inédites sur sa souffrance et qu'il a pu en outre les présenter sur scène lors d'un spectacle ; c'est au sein d'un atelier musical qu'il a découvert le plaisir de jouer d'un instrument qui demande des mouvements répétitifs de la main quasi-onanistes, que l'on percevrait volontiers comme une « sublimation de [ses] compulsions autocalmantes » [43]; c'est la dynamique groupale de l'atelier de parole qui lui a permis de s'affranchir des confidences bordées d'identifications massives de type spéculaire que la psychothérapie duelle lui avait fait expérimenter ; c'est dans l'espace d'accueil informel « matriciel » du Centre qu'il a rencontré l'hospitalité inconditionnelle et la tolérance envers toutes les singularités, qui l'ont aidé à mieux intégrer la sienne. C'est enfin la vie pétillante, trépidante et vibrante du Collectif qui l'a conduit à formuler le vœu de rester au Centre pendant «neuf mois » avant de déployer ses ailes dans un ailleurs autonome idéalisé, vœu que l'on pourrait lire en filigrane comme l'espoir d'une nouvelle naissance.

Si cette aspiration de Léonard paraît illusoire au vu de sa grande fragilité, sans doute consécutive à une démarche inconsciente conjointe de ses deux parents pour l'abandonner ou lui interdire de naître psychiquement ${ }^{17}$, elle témoigne néanmoins des effets du changement induits par la psychothérapie institutionnelle. Depuis dix mois, ses addictions ne l'ont « rappelé à l'ordre » qu'à deux reprises, à la suite d'événements extérieurs liés à son père. Ces rechutes exceptionnelles rappellent, d'une part, la précarité de son enveloppe psychique, d'autre part, elles font preuve du rôle contenant et pare-excitant de l'institution, du moins tant que des incidents de la réalité externe trop effractantes ne viennent pas le désorganiser. S'il demeure en proie à un ennui inquiétant pendant le weekend, lorsque le Centre est fermé, l'astreinte permanente lui permet de tenir jusqu'au lundi où il retrouvera le groupe sacralisé de ses «garde-fous » : les activités collectives, l'effervescence de la salle d'accueil et une équipe soignante désirante, tous ensemble incarnant un Surmoi institutionnel, un « Surmoi-Dulcinée » [45] tendre et bienveillant, capable de réguler

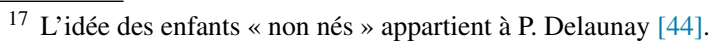


la jouissance erratique de Léonard et d'assouplir son « Surmoi-Célestine » [45] féroce, haineux et dévastateur.

Tout conduit à penser que le transfert plurifocal, la multiréférentialité et la transversalité de la psychothérapie institutionnelle possèdent la valeur de néo-objets vivants, transitionnels et humanisants, susceptibles de pacifier l'imago des parents empoisonnants, ainsi que de prendre la place des objets toxiques non humains fétichisés. S'il est toujours délicat d'envisager les perspectives d'avenir en clinique, on peut espérer que Léonard continuera à s'épanouir au sein du Collectif. Il conservera, certes, sa vulnérabilité, ses tentations et ses peurs de rechute, mais tempérées par l'assurance que, quoiqu'il lui arrive, il ne sera pas abandonné par sa nouvelle famille plurielle.

\section{Déclaration de liens d'intérêts}

L'auteur déclare ne pas avoir de liens d'intérêts.

\section{Références}

[1] Green A. Révélations de l'inachèvement : À propos du carton de Londres de Léonard de Vinci. Paris: Flammarion; 1992.

[2] Toubiana EP. La passion du risque : James Bond 007. Topique 2007;2(107):17-30.

[3] Chaperot C. Formes de transfert et Schizophrénie. Toulouse: Érès; 2014.

[4] Oury J. Les séminaires de la Borde, 1996/1997. Nîmes: Champ social; 1998.

[5] Oury J. Psychiatrie et psychothérapie institutionnelle : traces et configurations précaires. Lecques: Éd. du Champ social; 2001.

[6] Chaperot C, Altobelli A. Psychiatrie et ambiance. Evol Psychiatr 2014;3(79):541-55.

[7] Luca-Bernier (de) C. L'Entre-Corps : la Symbiose partielle dans l'approche des psychoses en psychothérapie institutionnelle. Vanves: MJW Fédition; 2013.

[8] Gauthier S, Joubert C, Souffir V. Traitements institutionnels. In: Kapsambelis V, editor. Manuel de psychiatrie clinique et psychopathologique de l'adulte. Paris: PUF; 2012. p. 1033-74.

[9] Oury J. Le Collectif : le Séminaire de Sainte-Anne. Nîmes: Champ social; 2005.

[10] Ferenczi S. Confusion de langue entre les adultes et l'enfant : le langage de la tendresse et de la passion ; 1932. Psychanalyse, Euvres Complètes 1927-1933, IV. Paris: Payot; 1982. p. 125-35.

[11] Chemla P. Neutralité malveillante. La Clin Lacan 2009;15:43-59.

[12] Faugeras P. La folie en partage, avec Gaetano Benedetti. Evol Psychiatr 2016;81:1-16.

[13] Toubiana EP. Introduction. In: Toubiana EP, editor. Addictologie Clinique. Paris: PUF; 2011. p. 1-20.

[14] Assoun PL. Psychanalyse et addictions. In: Toubiana EP, editor. Addictologie Clinique. Paris: PUF; 2011. p. 21-104.

[15] Freud S. Deuil et mélancolie; 1917 [1915]. In: Métapsychologie. Paris: Gallimard; 1968. p. 145-71.

[16] Benedetti G. Séminaires cliniques sur l'hystérie. Toulouse: Érès; 2012.

[17] Winnicott WD. Mirror-Role of Mother and Family in Child Development; 1967. In: Playing and Reality. London: Routledge Classics Edition; 2005. p. 149-59.

[18] Green A. La mère morte ; 1980. In: Narcissisme de vie. Narcissisme de mort. Paris: Éditions de Minuit; 2007. p. 247-83.

[19] M’Uzan (de) M. Les esclaves de la quantité. In: La Bouche de l'Inconscient : Essai sur l'interprétation. Paris: Gallimard; 1994. p. 155-68.

[20] Fain M. La Nuit, le Jour : Essai sur le fonctionnement mental. Paris: PUF; 1975.

[21] Szwec G. Les procédés autocalmants par la recherche répétitive de l'excitation. Rev Fr Psychosom 1993;4:27-51.

[22] Winnicott DW. Transitional Objects and Transitional Phenomena; 1953. In: Playing and Reality. London: Routledge Classics Edition; 2005. p. 1-34.

[23] Duparc F. Traitement de noyaux fétichiques, autistiques, ou autocalmants ? Conférence d'introduction à la psychanalyse de l'enfant du 17 mars, 2005. Cure psychanalytique de l'addiction [en ligne]. In: Société Psychanalytique de Paris; 2005 [consulté le $1^{\text {er }}$ juin 2015] http://www.spp.asso.fr/wp/?p=5867.

[24] Green A. Didier Anzieu vu par son jumeau imaginaire : À propos de Le Penser, du Moi-peau au Moi pensant. Le Carnet PSY 2007;5(118):33-7. 
[25] Lesourne O. La Genèse des addictions : Essai psychanalytique sur le tabac, l'alcool et les drogues. Paris: Presses universitaires de France; 2007.

[26] Khan M. Le concept du traumatisme cumulatif. In: Le Soi Caché. Paris: Gallimard; 1976. p. 69-87.

[27] Freud S. Lettres à Fliess, 1897-1904. Éd. complète. Paris: PUF; 2006. p. 365-8.

[28] Assoun PL. L'intoxication est un effort de faire sans l'autre. In: Assoun PL, editor. Les Nouvelles Addictions. Paris: Scali; 2007. p. 7-16.

[29] Chasseguet-Smirgel J. La Maladie d'idéalité : Essai psychanalytique sur l'idéal du moi. Paris: Montréal : L'Harmattan; 1999.

[30] Klein M. La Psychanalyse des enfants. Paris: PUF; 1993.

[31] Donnet JL, Surmoi. In: Mijolla (de) A, editor. Dictionnaire international de la psychanalyse, 1. Paris: Hachette Littératures; 2005.

[32] Freud S. Le Moi et le Ça ; 1923. In: Essais Psychanalytiques. Paris: Payot; 1981. p. 220-75.

[33] Green A. Le narcissisme de mort : structure ou état ; 1966-1967. In: Narcissisme de vie. Narcissisme de mort. Paris: Éditions Gallimard; 2007. p. 88-147.

[34] Lacan J. Idéal du Moi et Moi idéal ; 1949. In: Le Séminaire. Livre I : Les Écrits techniques de Freud $1953-1954$. Paris: Seuil; 1975. p. 205-27.

[35] Lacan J. Remarque sur le rapport de Daniel Lagache : «Psychanalyse et structure de la personnalité » ; 1954. In: Écrits, 2. Paris: Seuil; 1999. p. 124-66.

[36] Lehmann JP. Une clinique du transfert et de ses bords. In: Chemla P, editor. Aux limites du sujet. Ramonville, Saint-Agnès: Érès; 2006. p. 25-35.

[37] Le Poulichet S. Toxicomanies et Psychanalyse : les Narcoses du désir. Paris: Presses universitaires de France; 2011.

[38] Wilden A. System and Structure: Essays in communication and exchange. 2nd. ed. London: Routledge; 2001.

[39] Bion WR. Différenciation des personnalités psychotique et non psychotique ; 1958. In: Réflexion faite. Paris: PUF; 1983. p. 51-73.

[40] Czermak M. Patronymies : Considérations cliniques sur les psychoses. Toulouse: Érès; 2012.

[41] Chemama R. Clivage et Modernité. Toulouse: Érès; 2003.

[42] Nasio JD. Les Yeux de Laure : Nous sommes tous fous dans un recoin de notre vie. Nouv. éd. Paris: Payot \& Rivages; 2009.

[43] Smadja C. À propos des procédés autocalmants du Moi ; 1993. In: La Vie Opératoire : Études Psychanalytiques. Paris: PUF; 2001. p. 219-41.

[44] Delaunay P. Les Quatre Transferts. Paris: Fédération des Ateliers de psychanalyse; 2011.

[45] Macedo (de) HO. Lettres à une jeune psychanalyste. Paris: Stock; 2008. 